\title{
Prognosis and Early Prediction of Acute Kidney Injury in Critically Ill Children
}

\author{
Fatih Aygun ${ }^{1, *}$ \\ ${ }^{1}$ Cerrahpasa Medical Faculty, Istanbul University, Istanbul, Turkey \\ "Corresponding author: Cerrahpasa Medical Faculty, Istanbul University, Istanbul, Turkey. Tel: +90-5305534513, Email: faygun9@hotmail.com
}

Received 2018 August 18; Accepted 2018 September 06.

\begin{abstract}
Background: Acute kidney injury (AKI) is a serious clinical condition in children admitting to the pediatric intensive care unit. Beside having the risk of significant morbidity and mortality, AKI has also increased the risk for chronic kidney disease. However, there is paucity of data regarding AKI epidemiology in children. Herein, we aimed to describe the incidence, risk factors, and outcome of AKI in critically ill children.

Methods: The records of patients, who were hospitalized in our pediatric intensive care unit between October 2016 and March 2018 were investigated retrospectively. The relationship between AKI and the need of invasive mechanical ventilation and non-invasive mechanical ventilation, inotropic and nephrotoxic drug use, initial laboratory findings, continuous renal replacement need, pediatric risk of mortality-III score (PRISM-III), duration of hospitalization, and mortality rate were investigated.

Results: Totally, 447 patients were found to have been included in the study. The AKI developed in 111 patients. The sepsis was the most common underlying disease with 27 patients (24.3\%). There was a statistical significance between development of AKI and the need of invasive mechanical ventilation, inotropic and nephrotoxic drug use, PRISM-III score, continuous renal replacement need, plasma exchange, blood component transfusion, duration of hospitalization, and mortality.

Conclusions: The AKI is associated with prolonged hospitalization and increased mortality. AKI should be considered as a medical emergency that should be diagnosed and managed accurately.
\end{abstract}

Keywords: Pediatric Intensive Care Unit, Acute Kidney Injury, Hemodialysis, Prognosis

\section{Background}

Acute kidney injury (AKI) is among the common causes that increase mortality and morbidity in the pediatric intensive care unit (ICU). Beside, being an independent risk factor in the prognosis of the critically ill patient, AKI is also associated with increased health charges due to the need of dialysis and prolonged hospitalization $(1,2)$. Despite the improvement in renal replacement therapies and treatment modalities in intensive care, the mortality of AKI is still high, ranging from $9 \%$ to $67 \%(3,4)$. The early recovery of renal functions shortens the recovery and improves the outcome of patients with AKI. To prevent the AKI, the primary aim must be to determine the underlying risk factors and to provide the proper protective treatment strategies during and before admission to ICU. The epidemiology and the outcomes of AKI is well-defined in adults, however,the studies are limited in children, although the incidence of AKI can be as high as $83 \%$ (5).

Herein, we aimed to determine the incidence and to identify the causes, prognostic risk factors, and the outcome of AKI in pediatric intensive care unit (PICU).

\section{Methods}

\subsection{Study Design}

We performed a retrospective analysis of records of all patients admitted to PICU between October 2016 and March 2018. We have a tertiary, multidisciplinary PICU located in a training and research hospital in Istanbul, Turkey to which approximetly 20000 patients admit monthly during the winter season. Our PICU provides health-care for children from one month to 18 years, it has 12 beds, 11 incubators, two isolation rooms, and two Prismaflex $^{\mathrm{TM}}$ (Gambro, USA) hemofiltration machine. All catheters were attached by a single specialist and all intensive care follow up was done by the same specialist. 
2.2. Methods

Patients admitted with various causes of criticall illness in PICU of Okmeydani Research and Training Hospital between October 2016 and March 2018 were included in this study. The patients having the history of acute or chronic renal failure, having the dialysis for intoxication, metobolic disordes, tumor lysis and hemolytic uremic syndrome, patients staying less than 24 hours in the ICU, and who died in the first day of admission were excluded from the study.

The AKI was defined as oliguria (urine output $<0.5$ $\mathrm{mL}$ per kilogram of body weight per hour) and the elevated serum creatinine value for age or 1.5 fold increase in the baseline creatinine level in 24 hours. The estimated glomerular filtration rate (GFR) was calculated according to the original Schwartz formula. The blood gas analysis result comprises the serum creatinine and electrolyte levels as well in our PICU. The risk, injury, failure, loss of kidney function, and end-stage kidney disease (RIFLE) criteria was considered for patients at admission and during their stay in the PICU.

The age, gender, concomitant infection, the need of invasive or noninvasive mechanical ventilation, PRISM-III score, the usage of nephrotoxic and inotrophic drugs, laboratory findings at admission, blood component transfusion, plasma exchange, continuous renal replacement therapy (CRRT), the length of intensive care duration, and the mortality were the stated possible risk factors of AKI. The 29 drugs like acyclovir, amikacin, amphotericin b, vancomycin, and captopril were considered as nephrotoxic according to the Handbook of Pediatric \& Neonatal Dosage.

\subsection{Statistics}

Statistical analysis was performed using the IBM SPSS 20.0 (SPSS Inc., Chicago, IL, USA) package program. Continuous variables are expressed as mean \pm standard deviation and categorical variables are expressed as frequency (percentages). The Student $t$-test was used for continuous variables that have a normal distribution and the MannWhitney test for variables that did not have a normal distribution. Pearson's Chi-square and ANOVA test was used in the comparison of the categorical data between the groups. Univariate and multivariate binary logistic regression models were conducted to calculate odds ratio (OR) with 95\% confidence interval (CI) for PICU prognosis. A Pvalue of $<0.05$ was considered statistically significant.

\section{Results}

\subsection{Demographics}

Between October 2016 and March 2018 there were 469 children admitted to the ICU. A total of 447 patients were eligible for the present study. There were 22 patients that were excluded due to the fact that 17 patients had a known renal failure, two of them were diagnosed as hemolytic uremic syndrome, two of them died in the first 24 hour of hospitalization and one patient stayed shorter than 24 hours in the ICU. Of the 447 patients, 243 (54.4\%) were male, 204 (45.6\%) were female. The age distribution was between 1 month and 17 years, with a mean of $3.74 \pm 4.70$ years. The mean duration of hospitalization in the PICU was $7.32 \pm$ 10.29 days.

Overall, the respiratory failure, neurologic disorders, and the sepsis were the main causes of hospitalization. A total of 99 patients (22.1\%) recieved inotropic drugs and 168 (37.6\%) patients were used to recieve any nephrotoxic medication, 149 of which was amikacin. The mechanical ventilation (MV) was used in 122 (27.3\%) patients and non invasive mechanical ventilation (NIV) was efficient in ventilation of 204 (45.6\%) patients. A total of 50 (11.2\%) patients underwent CRRT. Plasma exchange was performed in 14 (3.1\%) patients. During the PICU stay a total of 14 (3.1\%) patients died (Table 1).

\subsection{Characteristics of AKI}

AKI was developed in 111 patients (24.8\%), 60 of patients (13.4\%) with AKI had increased creatinine levels, 13 patients (2.9\%) had two times the basal cretinine level, and 38 patients (8.5\%) had urine output $<0.5 \mathrm{~mL} / \mathrm{kg} / \mathrm{h}$. When the patients with the AKI were classified according to pRIFLE criteria (renal risk, injury, failure, loss of kidney function, end stage renal disease); 58 (52.2\%) patients had risk(R), 33 (29.7 $\%$ ) patients had injury (I), and 20 (18.0\%) patients had failure (F). The pRIFLE criteria and the outcome of the patients are shown in Table 2.

Totally, 50 patients (11.2\%) used to need CRRT, the underlying reason was AKI in 45 patients and intoxication in five patients. The patients who underwent CRRT were classified by the pRIFLE criteria, four (8.9\%) patients were with R category, 21 (46.7\%) patients were with I category, and 20 (44.4\%) patients in the F category received CRRT.

Sepsis was the most common underlying disease in AKI with 27 patients (24.3\%). PICU mortality rate was 3.1\% during the course of the study. All of the mortal cases developed AKI. A total of eight patients died due to septic 


\begin{tabular}{|c|c|c|}
\hline Number of Patients & All Patients in PICU $(n=447)$ & Patients Having AKI $(n=111)$ \\
\hline \multicolumn{3}{|l|}{ Gender } \\
\hline Male & $243(54.4)$ & $69(62.2)$ \\
\hline Female & $204(5.6)$ & $42(37.8)$ \\
\hline \multicolumn{3}{|l|}{ Reason for hospitalization } \\
\hline Respiratory System Disease & $159(35.6)$ & $19(17.1)$ \\
\hline Neurologic Diseasse & $73(16.3)$ & $9(8.1)$ \\
\hline Sepsis & $59(13.2)$ & $35(31.5)$ \\
\hline Cardiovascular Disease & $28(6.3)$ & $15(13.5)$ \\
\hline Intoxication & $45(10.1)$ & $1(0.9)$ \\
\hline Hematologic/Oncologic Disease & $16(3.6)$ & $10(9.0)$ \\
\hline Trauma/Surgery & $15(3.4)$ & $5(4.5)$ \\
\hline Others & $53(11.9)$ & $17(15.3)$ \\
\hline Age, $y$ & $3.74 \pm 4.70$ & $4.53 \pm 5.23$ \\
\hline Duration of stay in PICU, d & $7.32 \pm 10.29$ & $10.66 \pm 10.55$ \\
\hline Inotrophic medication & $99(22.1)$ & $61(55.0)$ \\
\hline Nephrotoxic medication & $168(37.6)$ & $40(36.0)$ \\
\hline Continious renal replacement therapy & $50(11.2)$ & $45(40.5)$ \\
\hline Invasive mechanical ventilation & $122(27.3)$ & $53(47.7)$ \\
\hline Noninvasive mechanical ventilation & $204(45.6)$ & $45(40.5)$ \\
\hline PRISM-III Score & $11.66 \pm 10.15$ & $15.20 \pm 12.34$ \\
\hline Plasma exchange & $14(3.1)$ & $11(10.0)$ \\
\hline Blood component transfusion & $160(35.8)$ & $70(63.1)$ \\
\hline Death & $14(3.1)$ & $13(11.7)$ \\
\hline
\end{tabular}

Abbreviations: SD, standard deviation; PICU, pediatric intensive care unit; PRISM, pediatric risk of mortality.

${ }^{a}$ Data are expressed as No. (\%) or mean \pm SD.

\begin{tabular}{lccc}
\hline Table 2. The pRIFLE Criteria and the Outcome of the Patients ${ }^{\mathrm{a}}$ & & \\
\hline & Rate $(\mathbf{n}=\mathbf{1 1 1})$ & CRRT $(\mathbf{n}=\mathbf{4 5})$ & Death $(\mathbf{n}=\mathbf{1 3})$ \\
\hline Risk & $58(52.2)$ & $4(8.9)$ & $0(0)$ \\
Injury & $33(29.7)$ & $21(46.7)$ & $2(15.4)$ \\
Failure & $20(18.0)$ & $20(44.4)$ & $11(84.6)$ \\
\hline
\end{tabular}

${ }^{\text {a }}$ Data are expressed as No. (\%).

shock and multiorgan failure, three patients had terminal state malignancy, one patient had congenital heart disease, one patient had sudden infant death, and one patient had brain edema secondary to metabolic disease. In total, 13 of the 14 patient who died used to recieve CRRT.

There was a statistically significant relationship between AKI and duration of hospitalization in the intensive care unit and mortality. P values were $\mathrm{P} \leq 0.001, \mathrm{P} \leq 0.001$. The mean duration of ICU stay was $10.66 \pm 10.55$ days for patients with AKI compared to $8.31 \pm 6.46$ days for those without AKI.

There was a statistically significant relationship between AKI and the age, gender, IMV support, inotropic and nephrotoxic drug use, PRISM-III scores, CRRT, blood component transfusion, and plasma exchange. $\mathrm{P}$ values were $\mathrm{P}=0.038, \mathrm{P}=0.045, \mathrm{P} \leq 0.001, \mathrm{P} \leq 0.001, \mathrm{P} \leq 0.001, \mathrm{P} \leq$ $0.001, \mathrm{P} \leq 0.001, \mathrm{P} \leq 0.001, \mathrm{P} \leq 0.001$, respectively (Table 3). There was no statistically significant difference in terms 
of concomitant infection, NIV, duration of IMV, and NIV. As compared in terms of laboratory levels at admission of ICU, there was a statistically significant relationship between AKI and platelet number and procalcitonin, C-reactive protein, serum magnesium, calcium, and lactate dehyrogenase levels. $\mathrm{P}$ values were $\mathrm{P} \leq 0.001, \mathrm{P} \leq 0.001, \mathrm{P} \leq 0.001, \mathrm{P}$ $=0.009, \mathrm{P}=0.036$, and $\mathrm{P}=0.042$, respectively (Table 3 ).

The odds ratios and relationship between prognostic factors and AKI were calculated by logistic regression models (Table 4). The odds ratio was 12.66 for plasma exchange and AKI. Inotrophic medication, blood component transfusion, hypomagnesemia, mechanical ventilation, and nephrotoxic medication were the parameters having high odds ratios.

\section{Discussion}

Acute kidney injury (AKI), previously called acute renal failure, is the reversible loss of kidney function characterised by decline in the glomerular filtration rate, increase in the blood concentration of creatinine as well as nitrogenous waste products, and deteriotation of fluid and electrolyte homeostasis $(6,7)$. AKI is associated with increased mortality, prolonged hospital stay, and increased hospital charges. The AKI is an independent risk factor in the worse prognosis of critically ill patients. In a recent study of 8260 patients, the mortality rate was $25.3 \%$ for the patients who developed AKI and 2.7\% for those who did not develop AKI (8).

The incidence of AKI has been reported to increase in critically ill patients over the past two decays. The RIFLE and Acute kidney injury network (AKIN) classifications are the two recently described standardized definitions of AKI, which provides physicians to be awake of the AKI $(9,10)$. The increase of the incidence may be due to the early recognition of AKI by the help of these new classification methods rather than the improvements in laboratory and dialysis opportunity (11). The two diagnostic criteria both use the serum creatinine and urine output levels rather than the underlying etiologic cause. The validity of two methods has been investigated in various studies. These criterias were found to be significantly associated with increased need for RRT, prolonged duration of recovery of renal functions, and the length of hospital stay and mortality. Even small increases in serum creatinine level $(\geq 0.3$ $\mathrm{mg} / \mathrm{dL}$ ) showed a significant effect on mortality of the critically ill patient $(5,12-14)$.

As a more updated definition, the KDIGO is also used in staging the AKI (15). There are many studies, both in adults and children, comparing the superiority of these classification methods. Bastin et al. compared the criteria in 1881 adults and reported that the AKIN and KDIGO better correlated with the mortality rather than RIFLE (16). In contrast, Fujii et al. reported that RIFLE and KDIGO indicated a better mortality differentiation than AKIN in 50000 adult patients (17). Zappitelli et al. studied the eligilibity of the criteria in children and reported the pRIFLE definition was more sensitive for AKI detection (18). However, three criteria have different advantages and there is not a clearly defined consensus regarding which criteria to prefer in children. The pRIFLE citeria was used in our study.

Different results had been reported regarding the incidence of the AKI in children in various studies. The highest result was in the study of Akcan-Arikan et al. with the $82 \%$ incidence according to pRIFLE criteria (5). In the present study, $24.8 \%$ of our patients developed AKI, similar to the reports of Slater et al. and Soler et al. who reported $23.7 \%$ and $27.4 \%$, respectively $(19,20)$. The pRIFLE criteria was used in both reports, which were the same as our study. The difference in reported rates of AKI may be due to the differences in profile of the study populations, the inclusion criteria, and the conditions as well as the enviroment of the ICU.

The frequent use of nephrotoxic drugs along with the solid organ and bone marrow transplantations and improved medical therapies in congenital heart diseases, sepsis, and septic shock may also have lead to the increase of AKI (21). Despite many recent developments in treatmant modalities and the usability of RRT in the ICU, the mortality and the morbidity of the AKI is still so high. On the other hand, though it is potentially reversible, AKI is an important source of chronic renal failure. Early identification of the factors affecting the prognosis and intervention with effective and rapid treatment approaches will contribute to decrease mortality and morbidity in AKI. Sepsis was the most important underlying cause of AKI. The mortality of patients was also significantly associated with AKI. The AKI increases the mortality 3.408 times according to logistic regression analysis. Plasma exchange was usually performed for patients with septic shock having thrombocytopenia associated multiorgan failure. Therefore, the risk of AKI was high in patients who used to need plasma exchange.

The younger age, sepsis, shock, need for mechanical ventilation, vasoactive drugs, and exposure to nephrotoxic drugs are the most commonly reported underlying risk factors of the AKI. The PRISM-III score, mechanical ventilation, the inotrophic and nephrotoxic medication, the length of hospitalization in ICU, CRRT, blood component 


\begin{tabular}{|c|c|c|c|}
\hline & AKI Developed $(\mathbf{n}=111)$ & AKI not Developed $(n=336)$ & $\mathbf{P}$ \\
\hline Gender & & & 0.038 \\
\hline Male & $69(62.2)$ & $174(51.8)$ & \\
\hline Female & $42(37.8)$ & $161(47.9)$ & \\
\hline Age, $y$ & $4.53 \pm 5.23$ & $3.50 \pm 4.49$ & 0.045 \\
\hline Invasive mechanical ventilation & $53(47.7)$ & $69(20.5)$ & $\leq 0.001$ \\
\hline Inotrophic medication & $61(55.0)$ & $38(11.4)$ & $\leq 0.001$ \\
\hline Nephrotoxic medication & $40(36.0)$ & $53(15.8)$ & $\leq 0.001$ \\
\hline Noninvasive mechanical ventilation & $45(40.5)$ & $159(47.3)$ & 0.145 \\
\hline Death & $13(11.7)$ & $1(0.0)$ & $\leq 0.001$ \\
\hline Duration in PICU & $10.66 \pm 10.55$ & $6.21 \pm 7.34$ & $\leq 0.001$ \\
\hline PRISM-III score & $15.20 \pm 12.34$ & $8.31 \pm 6.46$ & $\leq 0.001$ \\
\hline Duration of mechanical ventilation, $d$ & $11.75 \pm 19.25$ & $6.96 \pm 8.07$ & 0.070 \\
\hline Duration of noninvasive mechanical ventilation & $4.00 \pm 3.01$ & $3.75 \pm 3.99$ & 0.696 \\
\hline Concommitant Infection at admisssion & $78(70.3)$ & $225(66.9)$ & 0.314 \\
\hline$>7$ days hospitalization & $49(44.1)$ & $100(29.8)$ & 0.004 \\
\hline \multicolumn{4}{|l|}{ Laboratory findings at admission } \\
\hline Leucocyte number, /uL & $13501 \pm 9202$ & $13164 \pm 7292$ & 0.704 \\
\hline Procalcitonin, ng/mL & $30.08 \pm 38.78$ & $4.02 \pm 15.21$ & $\leq 0.001$ \\
\hline C-reactive protein, $\mathrm{mg} / \mathrm{L}$ & $74.06 \pm 96.61$ & $38.35 \pm 60.83$ & $\leq 0.001$ \\
\hline Platelet number, /uL & $262688 \pm 189984$ & $369612 \pm 170217$ & $\leq 0.001$ \\
\hline Magnesium, mg/dL & $2.04 \pm 0.56$ & $2.20 \pm 0.40$ & 0.009 \\
\hline Clorine, $\mathrm{mmol} / \mathrm{L}$ & $104.32 \pm 8.67$ & $101.68 \pm 6.15$ & 0.140 \\
\hline Sodium , mmol/L & $137.39 \pm 7.14$ & $137.72 \pm 5.47$ & 0.811 \\
\hline Calcium, mg/dL & $8.86 \pm 1.46$ & $9.40 \pm 0.86$ & 0.036 \\
\hline Alanine aminotransferase (ALT), U/L & $67.69 \pm 102.68$ & $64.22 \pm 223.39$ & 0.940 \\
\hline Lactate dehidrogenase (LDH), U/L & $644.81 \pm 398.52$ & $440.59 \pm 386.29$ & 0.042 \\
\hline Continious renal replacemet therapy & $45(40.5)$ & $5(1.5)$ & $\leq 0.001$ \\
\hline Blood component transfusion & $70(63.1)$ & $90(26.8)$ & $\leq 0.001$ \\
\hline Plasma exchange & $11(10.0)$ & $3(0.09)$ & $\leq 0.001$ \\
\hline
\end{tabular}

Abbreviations: AKI, acute kidney injury; PICU, pediatric intensive care unit; PRISM, pediatric risk of mortality.

transfusion, and plasma exchange were the parameters that increased the frequency of AKI in our report. We also experience statistical difference in terms of age and the gender in development of AKI the same as the report of Mehta et al. and Willims et al. who demostrated the younger age and Gupta et al. who demonstrated female gender as risk factors of the AKI (22-24). Herein, male gender was a risk factor of AKI as Al-Jboor et al. declares (25).

The relationship between the need for mechanical ventilation and renal functions had been studied in many studies. The decrease in the cardiac output and renal blood flow during the MV is already known. The vasopressor usage and the MV were reported to lead to AKI in a study involving 60338 critically ill patients in Taiwan (26). In an other retrospective study, prolonged hospitalization time in ICU, prolonged MV usage, and increase in mortality were reported to be related with the AKI (27). Same as the previous reports, we also determined the usage of inotropic drugs and the MV were more common in patients who developed AKI. When logistic regression analysis was performed, inotrophic drug usage increased 5.163 times and mechanical ventilation support increased 3.523 times. 


\begin{tabular}{|c|c|c|c|}
\hline Risk & PValue & Odds Ratio & 95\% CI \\
\hline Gender & 0.044 & 1.742 & $1.016-2.988$ \\
\hline Concomitant infection & 0.544 & 1.156 & $0.725-1.842$ \\
\hline Mechanical ventilation & $\leq 0.001^{* *}$ & 3.523 & $2.231-5.563$ \\
\hline Noninvasive mechanical ventilation & 0.243 & 0.771 & $0.498-1.193$ \\
\hline Inotrophic medication & $\leq 0.001^{* *}$ & 5.163 & $2.664-10.009$ \\
\hline Blood component transfusion & 0.954 & 1.026 & $0.432-2.436$ \\
\hline Plasma exchange hiponatremi & $\leq 0.001^{* *}$ & 12.660 & $3.461-46.307$ \\
\hline Nephrotoxic medication & $\leq 0.001^{* *}$ & 2.987 & $1.837-4.857$ \\
\hline Hypomagnesemia & $\leq 0.001^{* *}$ & 4.705 & $2.474-8.947$ \\
\hline Hypocalcemia & $\leq 0.001^{* *}$ & 4.931 & $2.991-8.130$ \\
\hline Hospitalization more than 7 days & 0.075 & 1.829 & $0.941-3.555$ \\
\hline Mortality & $\leq 0.001^{* *}$ & 3.408 & $0.003-0.175$ \\
\hline
\end{tabular}

Abbreviation: $\mathrm{CI}$, confidence interval.

However, we did not demonstrate any statistical correlation between the development of AKI and NIV use.

The sepsis is a well-established risk factor for the development and poor prognosis of AKI. Severe AKI is reported nearly in $20 \%$ of patients presenting with sepsis. The mortality of the septic AKI is reported to be three times higher when compared with nonseptic AKI in adults (28).

The sepsis associated AKI is also assumed to proceed chronic renal failure more frequently (28). We could not determine any statistically significant relationship between the accompanying infection and the development of AKI, however, initial procalcitonin and CRP levels at admission to PICU were higher in patients with AKI. The odds ratio was 1.156 for concomitant infection.

The nephrotoxic drugs had been reported to increase the frequency of AKI in several studies (19). They are established as the third most common cause of the AKI in PICUs. We had to use 168 nephrotoxic drugs (37.6\%) during the course of the study and the rate of the AKI was more in these patients $(\mathrm{P} \leq 0.001)$. Consistent with the other studies, the rate of the AKI was more common in patients who used amikacin and vancomycin. After this study, we started to be more aware of using nephrotoxic drugs in our clinic.

The duration of the ICU need was two times more in patients who developed AKI. Chertow et al. reported that each $0.5 \mathrm{mg} / \mathrm{dL}$ increase in creatinine level has prolonged the ICU need day by approximately 3.5 days (29). The prolonged hospitalization is known to increase the mortality and the hospital charges, especially since the hemodialysis and RRT increase the hospital costs considerably. In the current study, patients who developed AKI had a longer duration of hospitalization in ICU ( $\mathrm{P} \leq 0.001)$. CRRT, plasma exchange and blood componenet transfusion were also more common in patients with AKI.

Alves and colleagues reported in their study of $232 \mathrm{pa}-$ tients that hypomagnesemia is an independent risk factor for non-recovery of the renal function in critically ill patients with acute kidney injury (2). We also determined a significant relationship between hypomagnesemia and deterioration of renal functions. The AKI and CRRT were more common in patients with hypomagnesemia. Lastly, there was a statistically significant relationship between AKI and the initial platelet, calcium, and lactate dehidrogenase levels.

In conclusion, we tried to emphasize that AKI is a common clinical condition among the critically ill children. AKI is also closely related with the mortality and prolonged hospitalization. It is important to early recognize the risk factors leading to AKI and timely intervente to reduce hospital cost and patient mortality of AKI.

There are some limitations of our study. First, our study is a retrospective and single-centered study. Second, we do not have the cardiovascular surgery, bone marrow, and solid organ transplantation units in our hospital. Third, we only used the pRIFLE citeria and did not compare it with others, however, an apparent consensus regarding to which criteria to use in AKI definition in children is not yet notified. There is a need for prospective and multicenter studies involving more patients to define incidence and the prognostic factors of AKI in PICU. 


\section{Footnotes}

Authors' Contribution: Fatih Aygun: conception and design of the study, acquisition of data, analysis and interpretation of data, literature review and revision of manuscript. Fatih Aygun: design and writing of the study with literature review.

Ethical Considerations: Ethical approval for the study was provided by Local Committee (2018/847).

\section{References}

1. Bailey D, Phan V, Litalien C, Ducruet T, Merouani A, Lacroix J, et al. Risk factors of acute renal failure in critically ill children: A prospective descriptive epidemiological study. Pediatr Crit Care Med. 2007;8(1):2935. doi: 10.1097/01.pcc.0000256612.40265.67. [PubMed: 17251879].

2. Alves SC, Tomasi CD, Constantino L, Giombelli V, Candal R, Bristot Mde L, et al. Hypomagnesemia as a risk factor for the non-recovery of the renal function in critically ill patients with acute kidney injury. Nephrol Dial Transplant. 2013;28(4):910-6. doi:10.1093/ndt/gfs268. [PubMed: 22764195].

3. Palmieri T, Lavrentieva A, Greenhalgh D. An assessment of acute kidney injury with modified RIFLE criteria in pediatric patients with severe burns. Intensive Care Med.2009;35(12):2125-9. doi:10.1007/s00134009-1638-6. [PubMed: 19756500]. [PubMed Central: PMC2779390].

4. Kendirli T, Ekim M, Ozcakar ZB, Yuksel S, Acar B, Ozturk-Hiismi B, et al. Renal replacement therapies in pediatric intensive care patients: Experiences of one center in Turkey. Pediatr Int. 2007;49(3):345-8. doi: 10.1111/j.1442-200X.2007.02376.x. [PubMed: 17532833].

5. Akcan-Arikan A, Zappitelli M, Loftis LL, Washburn KK, Jefferson LS, Goldstein SL. Modified RIFLE criteria in critically ill children with acute kidney injury. Kidney Int. 2007;71(10):1028-35. doi: 10.1038/sj.ki.5002231. [PubMed: 17396113].

6. Andreoli SP. Acute kidney injury in children. Pediatr Nephrol. 2009;24(2):253-63. doi: 10.1007/s00467-008-1074-9. [PubMed: 19083019]. [PubMed Central: PMC2756346].

7. Mak RH. Acute kidney injury in children: The dawn of a new era. Pediatr Nephrol. 2008;23(12):2147-9. doi: 10.1007/s00467-008-1014-8. [PubMed: 18936981].

8. Sanchez-Pinto LN, Goldstein SL, Schneider JB, Khemani RG. Association between progression and improvement of acute kidney injury and mortality in critically ill children. Pediatr Crit Care Med. 2015;16(8):703-10. doi: 10.1097/PCC.0000000000000461. [PubMed: 26132741].

9. Bellomo R, Ronco C, Kellum JA, Mehta RL, Palevsky P; Acute Dialysis Quality Initiative Workgroup. Acute renal failure - definition, outcome measures, animal models, fluid therapy and information technology needs: The second international consensus conference of the acute dialysis quality initiative (ADQI) group. Crit Care. 2004;8(4):R204-12. doi: 10.1186/cc2872. [PubMed: 15312219]. [PubMed Central: PMC522841].

10. Mehta RL, Kellum JA, Shah SV, Molitoris BA, Ronco C, Warnock DG, et al. Acute Kidney Injury Network: Report of an initiative to improve outcomes in acute kidney injury. Crit Care. 2007;11(2):R31. doi: 10.1186/cc5713. [PubMed: 17331245]. [PubMed Central: PMC2206446].

11. Siew ED, Davenport A. The growth of acute kidney injury: A rising tide or just closer attention to detail? Kidney Int. 2015;87(1):4661. doi: 10.1038/ki.2014.293. [PubMed: 25229340]. [PubMed Central: PMC4281297].
12. Hoste EA, Clermont G, Kersten A, Venkataraman R, Angus DC, De Bacquer D, et al. RIFLE criteria for acute kidney injury are associated with hospital mortality in critically ill patients: A cohort analysis. Crit Care. 2006;10(3):R73. doi: 10.1186/cc4915. [PubMed: 16696865]. [PubMed Central: PMC1550961].

13. Ozcakar ZB, Yalcinkaya F, Altas B, Ergun H, Kendirli T, Ates C, et al. Application of the new classification criteria of the acute kidney injury network: A pilot study in a pediatric population. Pediatr Nephrol. 2009;24(7):1379-84. doi: 10.1007/s00467-009-1158-1. [PubMed: 19308461].

14. Sutherland SM, Byrnes JJ, Kothari M, Longhurst CA, Dutta S, Garcia P, et al. AKI in hospitalized children: Comparing the pRIFLE, AKIN, and KDIGO definitions. Clin J Am Soc Nephrol. 2015;10(4):55461. doi: 10.2215/CJN.01900214. [PubMed: 25649155]. [PubMed Central: PMC4386245].

15. Kidney Disease: Improving Global Outcomes (KDIGO) Acute Kidney Injury Work Group. KDIGO clinical practice guideline for acute kidney injury. Kidney Int Suppl. 2012;2(1):1-138. doi: 10.1038/kisup.2012.1. [PubMed Central: PMC4089619].

16. Bastin AJ, Ostermann M, Slack AJ, Diller GP, Finney SJ, Evans TW. Acute kidney injury after cardiac surgery according to risk/injury/failure/loss/end-stage, acute kidney injury network, and kidney disease: Improving global outcomes classifications. $J$ Crit Care. 2013;28(4):389-96. doi: 10.1016/j.jcrc.2012.12.008. [PubMed: 23743540].

17. Fujii T, Uchino S, Takinami M, Bellomo R. Validation of the kidney disease improving global outcomes criteria for AKI and comparison of three criteria in hospitalized patients. Clin J Am Soc Nephrol. 2014;9(5):848-54. doi: 10.2215/CJN.09530913. [PubMed: 24578334]. [PubMed Central: PMC4011454].

18. Zappitelli M, Moffett BS, Hyder A, Goldstein SL. Acute kidney injury in non-critically ill children treated with aminoglycoside antibiotics in a tertiary healthcare centre: A retrospective cohort study. Nephrol Dial Transplant. 2011;26(1):144-50. doi: 10.1093/ndt/gfq375. [PubMed: 20591815].

19. Slater MB, Gruneir A, Rochon PA, Howard AW, Koren G, Parshuram CS. Risk factors of acute kidney injury in critically ill children. Pediatr Crit Care Med. 2016;17(9):e391-8. doi: 10.1097/PCC.0000000000000859. [PubMed: 27472255].

20. Soler YA, Nieves-Plaza M, Prieto M, Garcia-De Jesus R, Suarez-Rivera M. Pediatric risk, injury, failure, loss, end-stage renal disease score identifies acute kidney injury and predicts mortality in critically ill children: A prospective study. Pediatr Crit Care Med. 2013;14(4):e18995. doi: 10.1097/PCC.ob013e3182745675. [PubMed: 23439463].[PubMed Central: PMC4238883].

21. Fortenberry JD, Paden ML, Goldstein SL. Acute kidney injury in children: An update on diagnosis and treatment. Pediatr Clin North Am. 2013;60(3):669-88. doi: 10.1016/j.pcl.2013.02.006. [PubMed: 23639662].

22. Mehta P, Sinha A, Sami A, Hari P, Kalaivani M, Gulati A, et al. Incidence of acute kidney injury in hospitalized children. Indian Pediatr. 2012;49(7):537-42. doi: 10.4103/0971-4065.177731. [PubMed: 22317984].

23. Williams DM, Sreedhar SS, Mickell JJ, Chan JC. Acute kidney failure: A pediatric experience over 20 years. Arch Pediatr Adolesc Med. 2002;156(9):893-900. doi: 10.1001/archpedi.156.9.893. [PubMed: 12197796].

24. Gupta S, Sengar GS, Meti PK, Lahoti A, Beniwal M, Kumawat M. Acute kidney injury in pediatric intensive care unit: Incidence, risk factors, and outcome. Indian J Crit Care Med. 2016;20(9):526-9. doi: 10.4103/0972-5229.190368. [PubMed: 27688628]. [PubMed Central: PMC5027745]. 
25. Al-jboor W, Almardini R, Al Bderat J, Frehat M, Al Masri H, Alajloni MS. Acute kidney injury in critically ill child. Saudi J Kidney Dis Transpl. 2016;27(4):740-7. doi: 10.4103/1319-2442.185236. [PubMed: 27424691].

26. Chang JW, Jeng MJ, Yang LY, Chen TJ, Chiang SC, Soong WJ, et al. The epidemiology and prognostic factors of mortality in critically ill children with acute kidney injury in Taiwan. Kidney Int. 2015;87(3):632-9. doi:10.1038/ki.2014.299. [PubMed: 25252027].

27. Volpon LC, Sugo EK, Consulin JC, Tavares TL, Aragon DC, Carlotti AP. Epidemiology and outcome of acute kidney injury according to pediatric risk, injury, failure, loss, end-stage renal disease and kidney disease: Improving Global outcomes criteria in critically ill children-
A prospective study. Pediatr Crit Care Med. 2016;17(5):e229-38. doi: 10.1097/PCC.0000000000000685. [PubMed: 26890198].

28. Fitzgerald JC, Basu RK, Akcan-Arikan A, Izquierdo LM, Pineres Olave $\mathrm{BE}$, Hassinger $\mathrm{AB}$, et al. Acute kidney injury in pediatric severe sepsis: An independent risk factor for death and new disability. Crit Care Med. 2016;44(12):2241-50. doi: 10.1097/CCM.0000000000002007. [PubMed: 27513354]. [PubMed Central: PMC5267552].

29. Chertow GM, Burdick E, Honour M, Bonventre JV, Bates DW. Acute kidney injury, mortality, length of stay, and costs in hospitalized patients. J Am Soc Nephrol. 2005;16(11):3365-70. doi: 10.1681/ASN.2004090740. [PubMed: 16177006]. 